\title{
Fabrication of circuits by multi-nozzle electrohydrodynamic inkjet printing for soft wearable electronics
}

\author{
Arshad Khan ${ }^{1, a)}\left(\mathbb{D}\right.$, Khalid Rahman², Shawkat Ali1, Salleem Khan ${ }^{1}$, Bo Wang ${ }^{1}$, Amine Bermak \\ ${ }^{1}$ Division of Information and Computing Technology, College of Science and Engineering, Hamad Bin Khalifa University, Qatar Foundation, Doha, \\ Qatar \\ ${ }^{2}$ Faculty of Mechanical Engineering, Ghulam Ishaq Institute of Engineering Sciences and Technology, Topi, Pakistan \\ a) Address all correspondence to this author. e-mail: arkhan4@hbku.edu.qa \\ Received: 10 January 2021; accepted: 25 March 2021; published online: 8 April 2021
}

Wearable electronic devices are evolving from current rigid configurations to flexible and ultimately stretchable structures. These emerging systems require soft circuits for connecting the various working units of the overall system. This paper presents fabrication of soft circuits by electrohydrodynamic (EHD) inkjet-printing technique. Multi-nozzle EHD printing head is employed for rapid fabrication of electric circuits on a wide set of materials, including glass substrate (rigid), flexible polyethylene terephthalate (PET) films, and stretchable thermoplastic polyurethane (TPU) films. To avoid the effects of substrate materials on the jettability, the proposed multi-nozzle head is equipped with integrated individual counter electrodes (electrodes are placed above the printing substrate). High-resolution circuits (50 \pm 5 $\mu \mathrm{m}$ ) with high electrical conductivity $\left(0.6 \Omega \square^{-1}\right)$ on soft substrate materials validate our well-controlled multi-nozzle EHD printing approach. The produced circuits showed excellent flexibility (bending radius $\approx 5 \mathrm{~mm}$ radius), high stretchability (strain $\approx 100 \%$ ), and long-term mechanical stability ( 500 cycles at $30 \%$ strain). The concept is further demonstrated with a soft strain sensor based on a multi-nozzle EHDprinted circuit, employed for monitoring the human motion (finger bending), indicating the potential applications of these circuits in soft wearable electronic devices.

\section{Introduction}

Inkjet printing, as an additive manufacturing technology, empowers fast prototyping of complex components for numerus applications, including electronics [1,2]. It offers several unique advantages for the fabrication of electronics as compared to conventional electronic fabrication techniques that employ several expensive and time-consuming steps such as lithographic patterning, chemical or physical vapor deposition of functional materials, and dry/wet etching to realize the device structures $[1,3]$. Over the years, many inkjet printers established with diverse actuation mechanisms such as piezoelectric, thermal, and aerosol are extensively employed in the electronics manufacturing. To retain high production output in these devices, printing heads usually composing of hundreds or even thousands of discrete nozzles are engaged [4-7].
The aforesaid printers are commercialized, yet, because of several inherent challenges of low resolution, head blockage, and overheating of functional inks, a novel inkjet-printing technique established on EHD atomization is an important research subject for numerous researchers in academia and industry [8-10]. Many investigators validated the viability of EHD inkjetprinting approach in realizing electronic devices by fabricating high-resolution metallic electrodes [11, 12], high-aspectratio (thickness to linewidth) structures $[13,14]$, self-powered stretchable sensors [15], stretchable photo-sensors [16], organic electronic memory devices [17], electrodes for solar cells [18], pads for LED display [19], and the source drain gate electrodes for thin-film transistors [20-23], having key features considerably smaller as compared to the printing nozzle [24, 25]. Despite all the advantages offered and initial success of EHD printing in fine patterning, its low throughput is an important limitation 
that has hindered its conceivable extensive applications in electronics manufacturing [26-28]. To overcome this deficiency and achieve a high throughput processing, multi-nozzle EHD printing approach has been primarily studied by few researchers [29-36]. However, these investigations are mainly concentrated on creating a stable jet and minimizing the deposition inaccuracy of the emitted jets/droplets. Also, the studied systems had multi-nozzle printing heads with discrete counter/ground electrodes, where the substrate during printing is placed in-between the terminals of the electric field. As a result, the jettability of the process is affected by the properties (dielectric constant) of the substrate material in addition to other printing parameters (applied voltage, ink flowrate, and distance between the printing nozzle and ground electrode). Furthermore, these investigations are limited to printing circuits on single substrate material, mostly rigid glass and, therefore, cannot be directly used to fabricate circuits on multiple substrates (without modifying the experimental parameters). The abovementioned limitations call for a reproducible multi-nozzle EHD printing approach that can rapidly produce high-resolution circuits on diverse substrate materials with consistent performance.

To make the EHD inkjet-printing technology commercially competitive for the additive manufacturing industry, we present an EHD inkjet-printing approach that employs a printing head comprising of five nozzles with integrated counter electrodes to simultaneously print on a variety of substrate materials without modifying the experimental parameters. To avoid the effects of substrate materials on the printing, the proposed multi-nozzle head is equipped with integrated individual counter electrodes, i.e., ring-shaped ground electrodes are engaged above the printing substrate. This multi-nozzle printing head consists of four main parts, i.e., poly(methyl methacrylate) (PMMA) holder, copper electrodes, glass nozzles, and copper ring extractors (ground electrodes), all of these integrated into a single structure. The concept is validated with a number of prototype circuits made of highly conductive silver inks on variety of substrate materials including rigid glass substrate, flexible PET film, and stretchable TPU foil. The printed circuits were further utilized in a soft strain sensor to demonstrate their practical potential in devices. The facile fabrication of these circuits shows that our multi-nozzle EHD inkjet-printing process is an effective approach to rapidly fabricate soft electronic structures and devices with high consistency.

\section{Results and discussion}

\section{Working principle of EHD printing}

EHD printing utilizes electric fields, rather than thermal or acoustic energy for the ink deposition. Figure 1a illustrates single-nozzle EHD inkjet-printing process, where a conducting ink in a needle/nozzle is electrified by means of high electric potential. A droplet stays spherical (Fig. S1a, Supporting Information), as the surface tension tends to minimize the interfacial energy. To reform this droplet from sphere to a cone, high electric potential (few $\mathrm{kV}$ ) is needed. By applying the required potential, the exterior of the ink meniscus as shown in Fig. S1b (Supporting Information) is mainly subjected to surface tension, hydrostatic force, and electrostatic forces. If the ink is assumed to be a perfect conductor, the electric field will act only normal to the surface of the ink, without having a tangential component, making the ink neutral, and as a result, the free charges will be confined in a very thin layer. However, in reality, the ink is not a pure conductor; therefore, the resultant electric polarization stress on the liquid surface has both the normal and tangential components. The normal electric stress disrupts the jet while the tangential electric stress moves the ink from the meniscus sides to the apex. When the tangential component is stronger than normal, a stable cone (typically known as Tyler cone) is formed, whose apex ejects a very small jet comparing to the size of the nozzle. This jet is then deposited onto a moving substrate to pattern a specified design $[37,38]$.

\section{Structure of the printing head}

In typical multi-nozzle EHD inkjet-printing process, the apex of the Tylor cone becomes marginally off-center particularly at the end nozzles, as a result of the interaction between the positively charged adjacent jets, making them outward tilted $[30,32]$. Two factors significantly affect this interaction between the adjacent nozzles. One of them is the space among the neighboring nozzles of the print head; generally, the interaction is inversely proportional to the spacing [39]. The other major factor is the composition (materials properties) of the printing nozzles. In-general, the usage of metallic needles/nozzles intensifies the interaction, comparing to the nozzles prepared with dielectric materials [31]. Therefore, we fabricated our proposed printing head (as schematically illustrated in Fig. 1b) with dielectric materials (glass nozzles and PMMA plastic holder), and designed it in such a way that each printing nozzle is isolated from the others to completely eliminate the interaction between the adjacent jets. Besides no interaction, the other key feature of our proposed head is the integrated electrodes. In most of the previously reported multi-nozzle EHD printing heads, discrete counter/ground electrodes have been used where the substrate is in-between the terminals of the electric field. As a result, the jetting parameters are also influenced by the material properties of the used substrate. In contrast, our printing head has the integrated ring-shaped counter/ground electrodes above the substrate, eliminating the disturbances in the electric field caused by the dielectric constant of the substrates and, hence, their effects on the jetting parameters. Therefore, in our approach, the jetting 
(a)

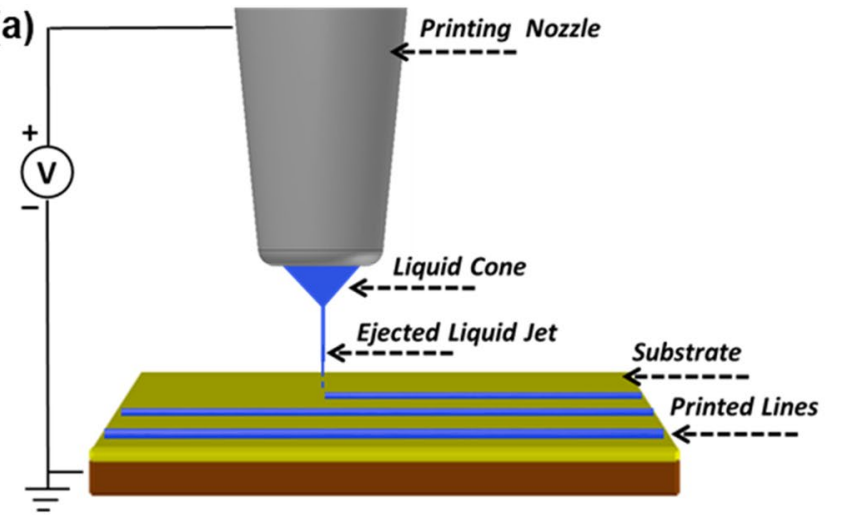

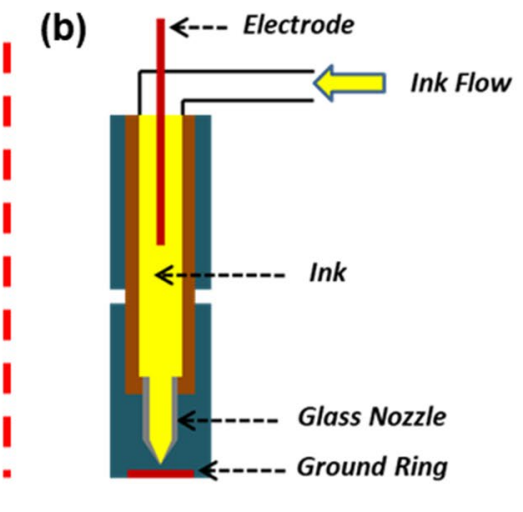

(c)

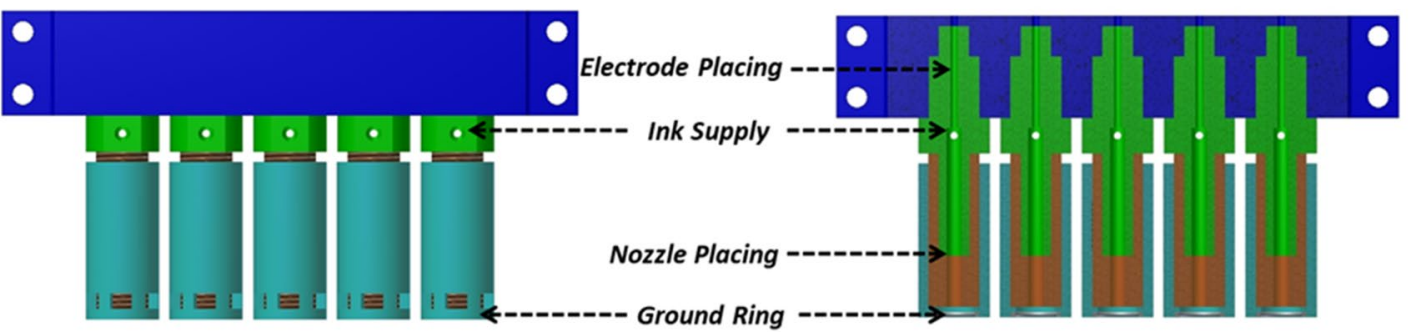

(d)
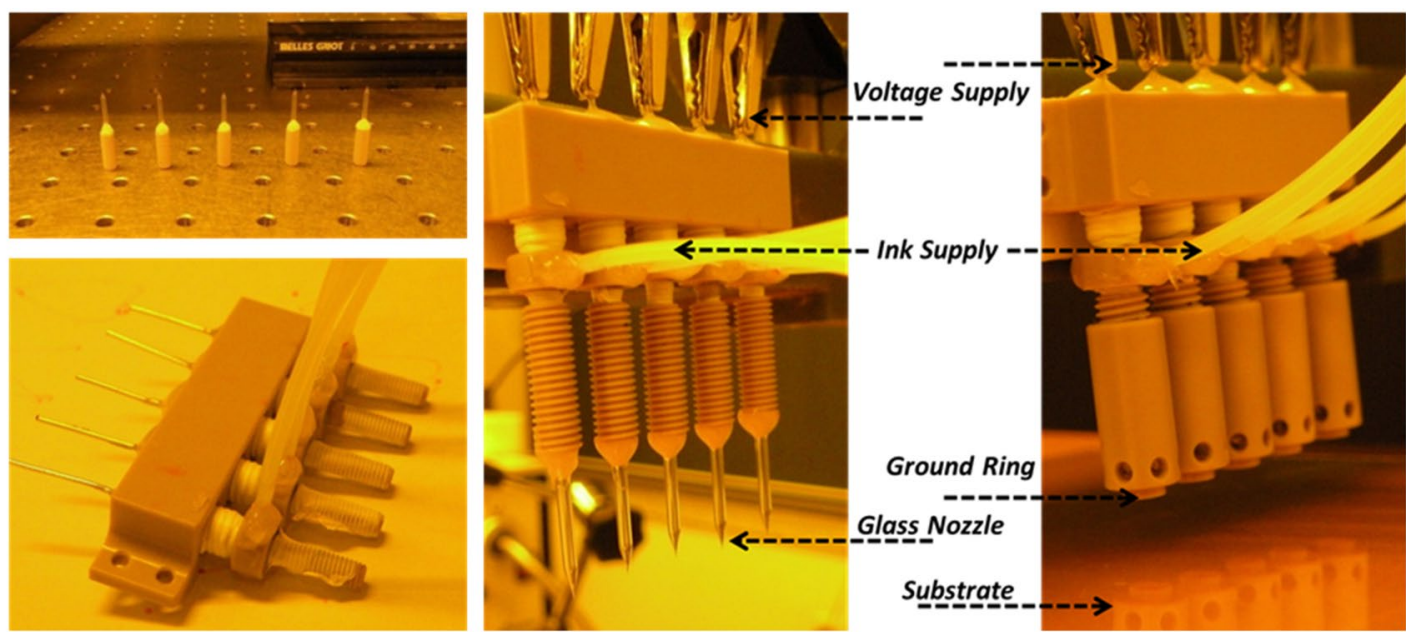

Figure 1: (a) Schematic illustration of the EHD inkjet-printing process. (b) Schematic illustrations of the proposed multi-nozzle EHD inkjet-printing head. (c) Schematic illustrations of the nozzles holder along with the cross-sectional view (right). (d) photographs of the multi-nozzle printing head, assembly (left) without (center), and with (right) integrated ground rings.

parameters require the optimization only once for every new printing ink. The schematic and photograph our printing head are displayed in Fig. 1c and d, respectively. Also the detailed drawings of the individual parts of the head are presented in Figs. S3-S7 (Supporting Information).

\section{Printed results}

First, experiments were executed with only one nozzle to optimize the nozzle size for the printings. Various nozzles sizes with diameter of $10 \mu \mathrm{m}, 15 \mu \mathrm{m}, 20 \mu \mathrm{m}, 25 \mu \mathrm{m}$, and $30 \mu \mathrm{m}$ were, respectively, tried for blockage through printing the conductive inks for a while. It was witnessed that the nozzles other than $30 \mu \mathrm{m}$ were got clogged after printing for some time. Accordingly, the $30 \mu \mathrm{m}$ nozzle size was chosen for printing the circuits. Next, experiments were performed to optimize the parameters for making a stable Tylor cone jet. It was observed that for silver nanoparticle ink, the minimum values of ink flow rate and applied voltage for stable cone-jet formation were found to be $20 \mu \mathrm{l} / \mathrm{h}$ and $3.5 \mathrm{kV}$, respectively. Applying these parameters, printing was performed by keeping the substrate speed at 20 $\mathrm{mm} / \mathrm{s}$. Figure 2a displays the scanning electron microscope (SEM) images of continuous silver nanoparticle-based circuits printed on the glass substrate via all five nozzles. As, the glass 
(a)
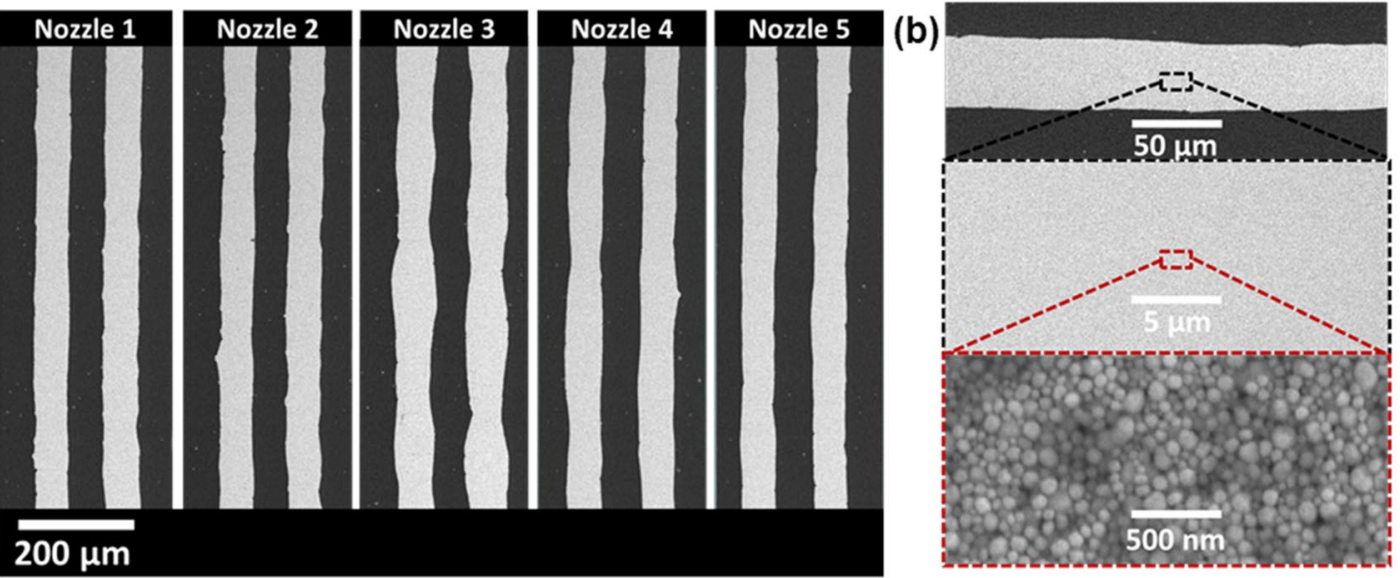

(c)
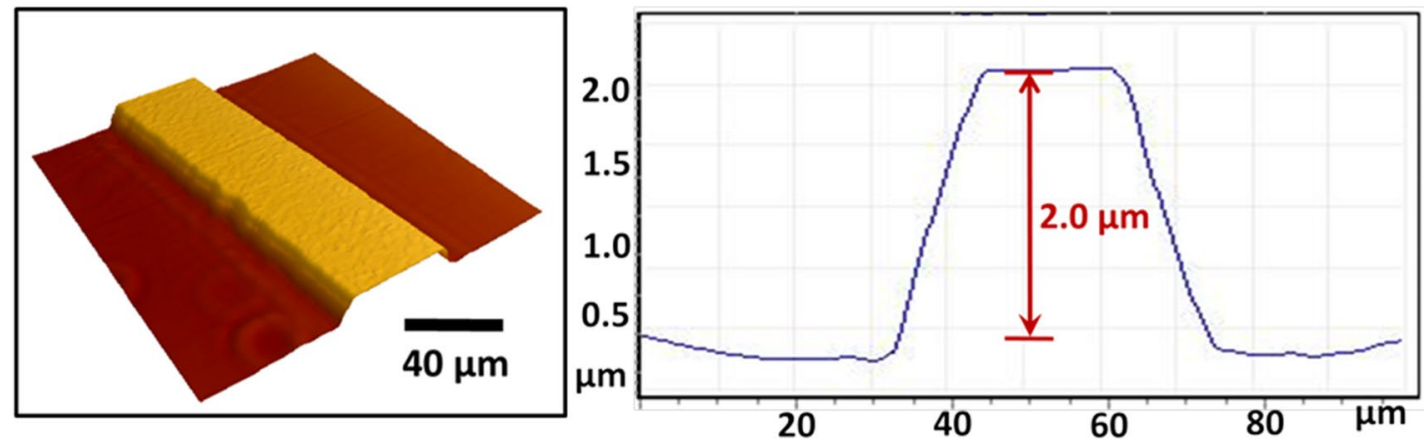

Figure 2: Morphological characterization of the porotype silver nanoparticles-based circuits on glass substrates. (a) SEM images of the circuits, simultaneously printed by our technique. (b) SEM images of the circuit at different resolutions. The lowermost inset displays the silver nanoparticlesbased cluster. (c) AFM images of a typical printed circuit. The cross-section image (right) displays its thickness.

substrate is hydrophilic in nature; therefore, the printed ink was spread out, making the size of the tracks wider than that of the jets. The minimum track width achieved is around $50 \mu \mathrm{m}$. This printing resolution can be further improved by making the glass substrate hydrophobic; however, in the present work, we did not treat the substrate for the printing resolution enhancement. Figure $2 b$ displays the SEM micrographs of a usual printed track at different magnifications. These images confirm that the nanoparticles are interlocked forming a three-dimensional cluster, which positively influence the conductivity of the printed circuits. Moreover, Fig. S2 (Supporting Information) presents the $\mathrm{X}$-ray diffraction (XRD) pattern of a characteristic silver nanoparticle-based circuit fabricated by our approach. The diffraction peaks correspond to the respective (111), (200), (220), and (311) planes, which confirms that the printed circuit is made of silver nanoparticles only. The atomic force microscope (AFM) image shown in Fig. 2c confirms that the height of a typical printed silver nanoparticle-based circuits is around $2 \mu \mathrm{m}$. After sintering at $250{ }^{\circ} \mathrm{C}$ for $30 \mathrm{~min}$, the resistance of the printed circuits on glass substrate was measured to be $0.4 \Omega \square^{-1}$ using standard 4 -point method. Due to its high curing temperature, electrically functional silver nanoparticle-based circuits cannot be realized on the PET and TPU substrate.
Organo-metallic inks have got special attention in printed electronics for their true-solution existence, where the metallic salts are completely dissolved in the solvents. This characteristic is especially desired for the EHD printing approach, as this can efficiently decrease the residue and blockage concerns rising from nanoparticles in the typical colloidal inks. Furthermore, a lower temperature for thermal decomposition is needed for these compounds, as an alternative to higher temperature required for curing of nanoparticles-based inks. These desirable attributions brand the organo-metallic inks reasonably attractive for EHD inkjet printing. Accordingly, optimizing the experimental parameters, printing of silver organo-metallic ink was performed on flexible PET films and stretchable TPU foils at an applied voltage of $3.9 \mathrm{kV}$ and ink flow rate of $20 \mu \mathrm{l} / \mathrm{h}$. Figure 3 displays the morphological characterization of the porotype organo-metallic silver circuit on PET substrate. Figure 3a presents the optical micrographs of the five silver tracks simultaneously printed on PET substrate. The minimum line width of around $100 \mu \mathrm{m}$ is achieved, which is larger than that of the silver nanoparticlesbased ink. This is due to the fact that organo-metallic ink spread out more as compared to silver nanoparticle-based ink. The SEM images as presented in Fig. 3b display the 
(a)

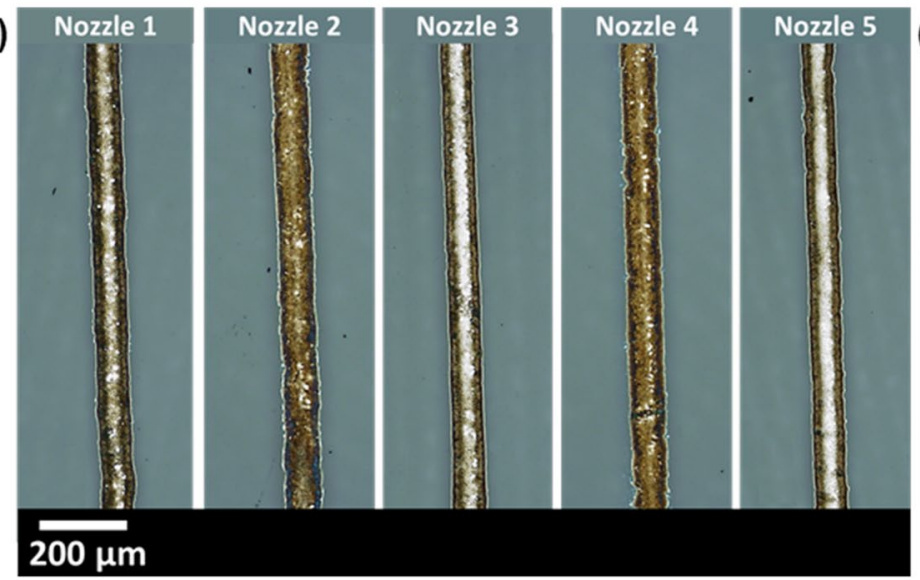

(b)

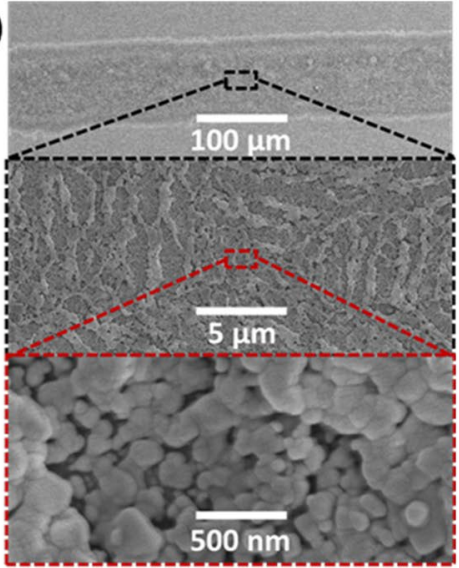

(c)
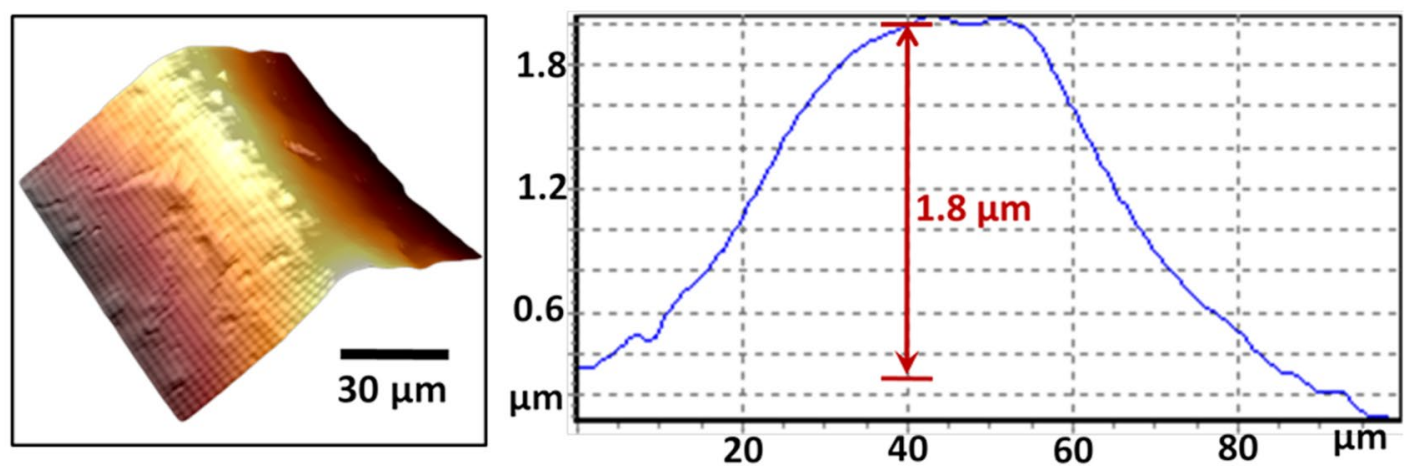

Figure 3: Morphological characterization of the porotype organo-metallic silver circuits on flexible PET substrate. (a) Optical microscope images of the circuits, simultaneously printed by our approach. (b) SEM images of the circuit at different resolutions. The bottom insets show densely packed crystallites. (c) AFM images of the printed line. The cross-section image (right) displays the thickness of the printed circuits.

microstructure of the printed tracks at various magnifications. These images reveal that the structure of the line is composed of densely packed crystallites. Minor porosity as can be noticed, arose because of the vaporous byproducts in the ink disintegration during the heat sintering. As these fumes exit the structure, silver metal film having a reasonable conductivity is produced. Yet, the porosity unavoidably decreases the electrical conductivity. The AFM image shown in Fig. $3 c$ confirms that the thickness of these printed circuits is around $1.8 \mu \mathrm{m}$, which are slightly thinner than those of silver nanoparticles-based circuits. After sintering at $100^{\circ} \mathrm{C}$ for $30 \mathrm{~min}$, the resistance of printed circuits were then recorded using standard 4-point method. The resistances of both the silver inks on different substrates are summarized in Table 1.

\section{Mechanical stability of the printed circuits}

Mechanical robustness against bending and stretching are important requirements for applications in soft electronics [40, 41]. Therefore, besides electrical performance measurements, flexibility and stretchability tests were performed on the printed circuits of organo-silver ink on PET film and TPU substrate, respectively. Figure $4 \mathrm{a}$ and $\mathrm{b}$ provides the flexibility test results of the samples (30 mm length and $1 \mathrm{~mm}$ line width) on PET film, under repeated compressive and tensile bendings. Figure 4a displays the change in resistance with the number of repetitive compressive bendings to a radii of $20 \mathrm{~mm}, 15 \mathrm{~mm}, 10 \mathrm{~mm}$, and $5 \mathrm{~mm}$. The outcomes show that for $20 \mathrm{~mm}$ and $15 \mathrm{~mm}$ bending radii, marginal resistance variation $\left(0.7 \Omega \square^{-1}\right)$ rises for 1000 bendings. However, for a $5 \mathrm{~mm}$ bending radius, the resistance variation is significant, yet, inside $300 \%$ of its initial value $\left(0.6\right.$ to $\left.1.8 \Omega \square^{-1}\right)$. Also, for tensile stress, resistance variations against the repetitive bendings are presented in Fig. 4b, indicating that for 1000 bending cycles to $20 \mathrm{~mm}, 15$
TABLE 1: Resistance (in $\Omega \square^{-1}$ ) of silver inks on different substrates.

\begin{tabular}{lcccc}
\hline \hline Ink/substrate & Glass (rigid) & Photo paper (ref) & PET (flexible) & TPU (stretchable) \\
\hline Silver nanoparticles ink & 0.4 & N/A & N/A & N/A \\
Silver organo-metallic ink & 0.5 & 0.8 & 0.6 & 0.7 \\
\hline \hline
\end{tabular}



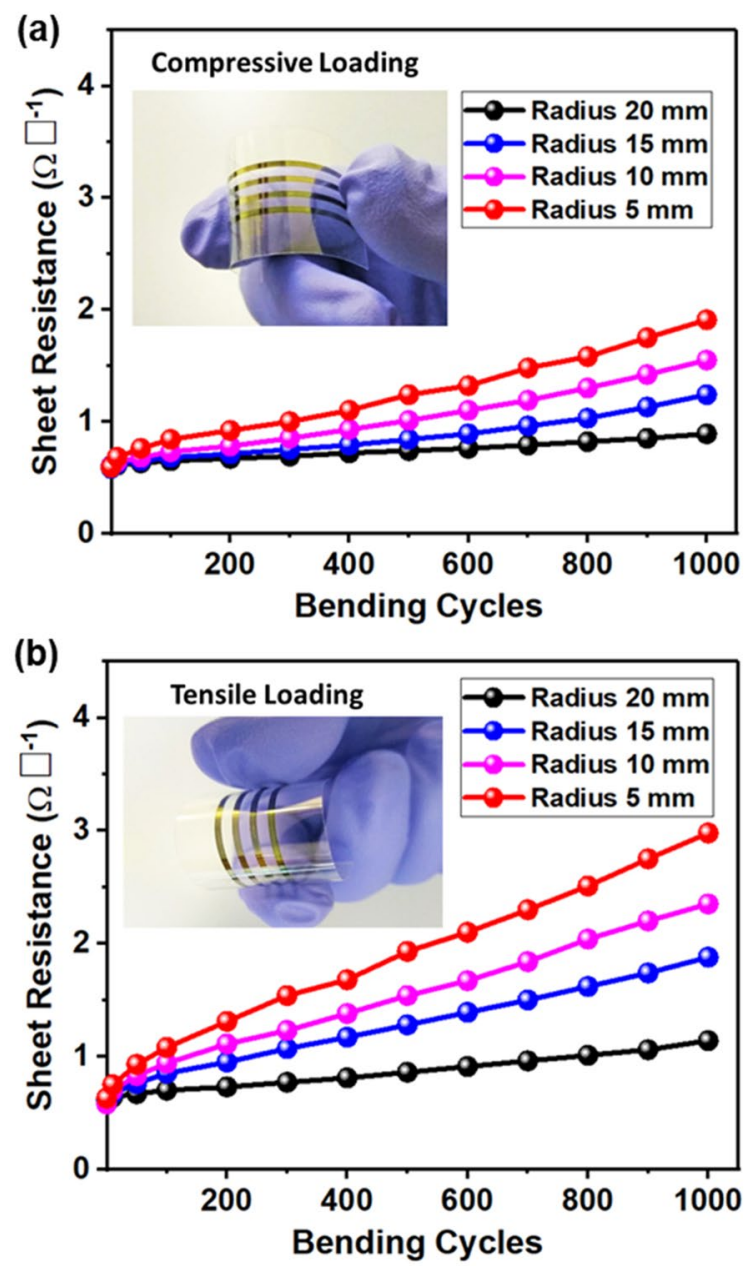

Figure 4: Mechanical flexibility of the printed circuits. (a) Resistance changes as function of repetitive bendings under compressive loading to radii of $20 \mathrm{~mm}, 15 \mathrm{~mm}, 10 \mathrm{~mm}$, and $5 \mathrm{~mm}$. (b) Resistance changes as function of repetitive bendings under tensile stress to radii of $20 \mathrm{~mm}, 15$ $\mathrm{mm}, 10 \mathrm{~mm}$, and $5 \mathrm{~mm}$.

$\mathrm{mm}, 10 \mathrm{~mm}$, and $5 \mathrm{~mm}$ radii, the resistances changed from 0.6 to $0.9 \Omega \square^{-1}$, from 0.61 to $1.45 \Omega \square^{-1}$, from 0.62 to $2.35 \Omega \square^{-1}$, and from 0.58 to $2.9 \Omega \square^{-1}$, respectively. These relatively greater resistances' rises apparently happen due to the thin-film cracking under tensile stress, revealing that these thin-metal films are extra susceptible to failure beneath tensile stress than compressive stress. However, additional experiments are needed to examine the failure phenomenon of these printed metal films applying different bending forces and their influences on the ultimate performance of the resulting soft wearable devices.

The metallic conductors are brittle which means their maximum tolerance to strain is extremely low [42-44]. To address this issue and enhance the stretchability of our printed silver circuits, we implement the substrate prestrain strategy in our experiments. Prestraining the elastomeric substrate before printing is one of the promising approach utilized earlier to enhance (a)

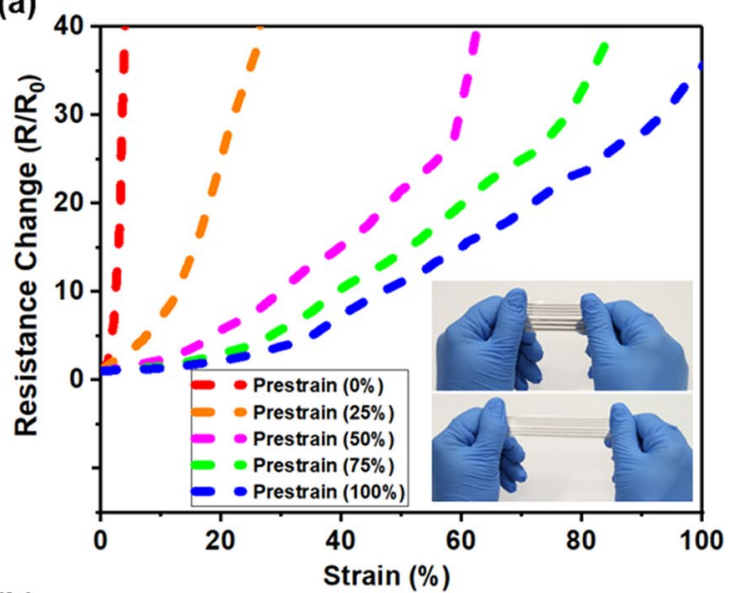

(b)

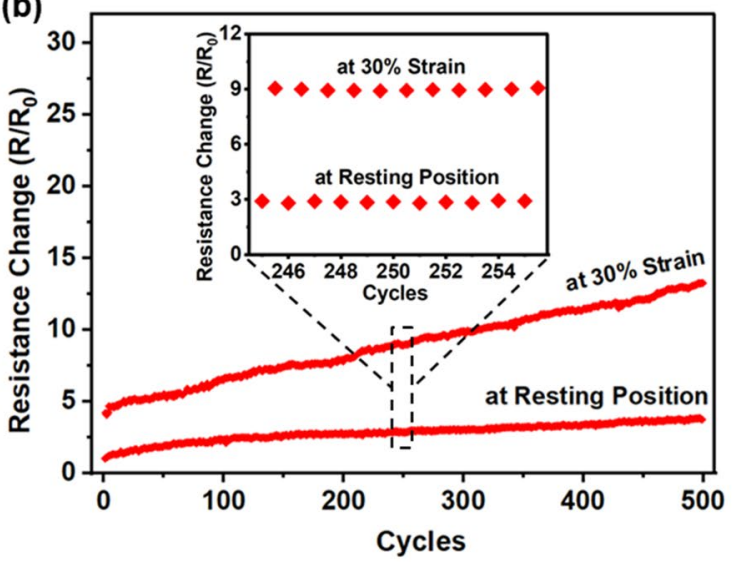

Figure 5: Mechanical stretchability of the printed circuits on TPU substrates. (a) Resistance variation with strain for different prestrains. (b) Strain behavior for 500 cycles for a multi-nozzle EHD-printed circuit on $100 \%$ prestrained substrate.

the stretchability of inkjet-printed traces [45-47]. Inspired from the idea, we incorporate this in our approach by using a rigid glass as a support to lock/keep the TPU film stretched while printing. The stretchability of the printed silver samples $(30 \mathrm{~mm}$ length and $1 \mathrm{~mm}$ line width) was examined in order to assess the effectiveness of the substrate prestraining strategy. The samples were printed on TPU substrate with different intensities of prestrain $(0 \% ; 25 \% ; 50 \% ; 75 \%, 100 \%)$. After thermal sintering, the samples are stretched by elongating them up to $100 \%$ of its initial length under uniaxial force, and the resistance values are plotted in Fig. 5a. It is evident that the silver line printed on unstretched substrate withstands only $\approx 4 \%$ strain. Nevertheless, if printed on a prestrained film, the samples are prominently more stretchable. Larger prestrain exhibits lower increase in resistance upon elongation, and vice versa. For instance, 25\% prestrain sample shows a resistance increase of 10 -folds at $12 \%$ strain, while the $50 \%$ prestrain sample reaches 10 -folds increase in resistance at $30 \%$ strain. Similarly, the $100 \%$ prestrain sample displays the best stretchability by reaching the 10 -folds increase 
at $47 \%$ strain. These increases in the resistances with stretching are apparently originate due to the cracks in the silver film caused by the plastic deformation. It is notable to mention that the conductivity of the films fully recovers after the stress release, and thus, these printed patches can be readily employed as strain sensors. For sensing applications, larger prestrain samples are beneficial for recording large-scale physical activities of the human limbs (e.g., bending, torsion, and stretching), as they are more stretchable and, thus, offer a wider sensing range. On the other hand, lower prestrain patches are better for monitoring the small-scale delicate movements (e.g., pulse, swallowing, heartbeat, and facial expressions), as these are highly sensitive to the external mechanical stimuli.

Also, to examine the strength against cyclic stretching and releasing, the resistance of a $100 \%$ prestrained TPU sample was measured by stretching it up to $30 \%$ of its initial length under uniaxial force. As plotted in Fig. 5b, the baseline resistance amplified almost linearly up to 3.75 -folds after 500 cycles. Furthermore, the resistance change ratio within each cycle remained nearly constant, from originally 4.2 times to 3.5 times after 500 cycles. The sample stayed functional after 500 cycles, which demonstrates its good mechanical and electrical endurance. According to these outcomes, the stretchability of these circuits is superior to that of the other inkjet-printed circuits [47-49], and is sufficient for most targeted applications, such as in wearable devices [1] and electronic skins [50, 51], where systems are not needed to be exceedingly stretchable.

\section{Wearable soft strain sensor based on printed circuits}

Soft resistive strain sensors are receiving enormous attention because of their simple architecture and high sensitivity
$[2,52]$. These sensors are particularly advantageous in realtime monitoring of health, electronic skin, measuring human daily physical, and behaviors [53,54]. Like other soft wearable devices, high-quality resistive strain sensors need to have the capability of conformal integration with any arbitrary and moving soft clothing or skins to precisely sense the external mechanical stimulations for health monitoring and disease diagnosis [55]. Furthermore, these sensors must also have the high stretchability in order to monitor large-scale human physical activities such as stretching and bending movements of the human joints [56]. A resistive strain sensor was fabricated using a multi-nozzle EHD-printed silver nanoparticlebased strain gage (40-mm line length, $2-\mathrm{mm}$ line spacing, $1 \mathrm{~mm}$ line width). The strain gage was printed on the prestrained (50\%) TPU film and was deployed on the human finger after thermal sintering, as shown in Fig. 6a. Resistance values were continuously recorded while the sensor was actively being elongated by the joints as the finger was bent, as displayed in Fig. 6b. The softness and the deformability of the TPU film enable the printed patch to conform readily to the finger's non-planer contours. The relative resistance variation was plotted as the finger was repetitively bent and straightened, as shown in Fig. 6c. A distinctive relative resistance variation was observed in every cycle, sensing the fingerbending movement. Figure $6 \mathrm{c}$ displays resistance variation for few bending cycles only; however, similar trend was observed for large number of cycles. This is due to the fact that the sensor patch does not experience excessively high strains during the finger bending, making the device suitable to be used for long-term monitoring of the finger-bending movement. This simple example of sensing the finger bending by multi-nozzle
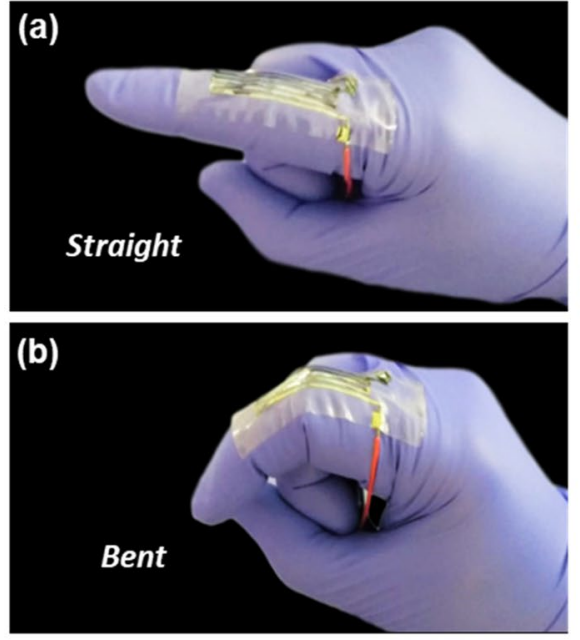

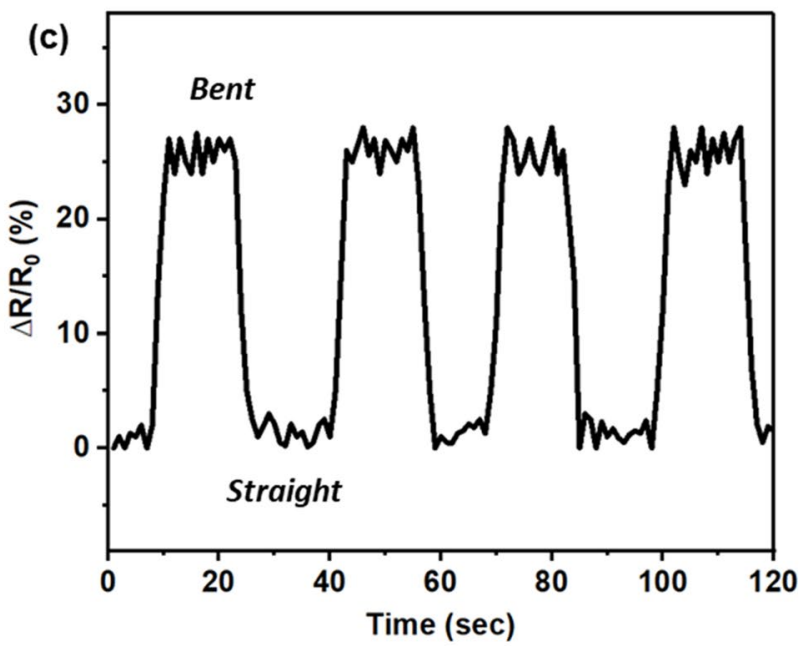

Figure 6: Soft strain sensor applied to monitor the finger movement. (a) Image of the strain sensor patch attached to a finger, showing it in unstretched form. (b) Image of the bent finger, showing the strain sensor in stretched form. (c) Relative resistance variation recorded with the movement of finger being repeatedly bent and straightened. 
EHD-printed patch proposes its enormous capability for sensing various other kinds of human movements.

\section{Conclusions}

In summary, we demonstrated a rapid EHD inkjet-printing approach that employs a printing head composing of five nozzles with integrated counter electrodes to simultaneously print on variety of substrate materials, including those of flexible and stretchable substrates. The simultaneous printing of high-resolution $(50 \pm 5 \mu \mathrm{m})$ circuits with high electrical conductivity $(0.6 \Omega$ $\square^{-1}$ ) on soft substrates confirms the successful implementation of our approach for rapid fabrication. The fabricated circuits exhibited high flexibility (bending radius $\approx 5 \mathrm{~mm}$ radius) and stretchability (strain $\approx 100 \%$ ), and long-term performance stability (500 cycles under $50 \%$ strain). Finally, a soft strain sensor was constructed with the printed circuits, which demonstrated excellent performance compared with the existing devices. It is believed that similar printed circuits, manufactured by our additive and rapid EHD inkjet-printing approach, will enable many commercial soft wearable electronic applications in the future.

\section{Materials and methods}

\section{Materials}

Commercial silver inks, colloidal ink containing silver nanoparticles (npk-020, NPK CO., Ltd), and silver particle-free solution (TEC-IJ-020, InkTec Elec.) were used for printing. The properties of inks are summarized in Table 2. Glass substrates (500$\mu \mathrm{m}$ thickness were used as a rigid substrate material. Flexible PET films (130- $\mu \mathrm{m}$ thickness) used in this study were purchased from Mitsubishi Paper Mills. Commercial TPU foils (Platilon ${ }^{\circledast}$ U073, Covestro) with thickness of $100-\mu \mathrm{m}$ were used as stretchable substrate material.

\section{Printing head fabrication}

The proposed printing head is composed of four parts, i.e., PMMA holder, glass capillaries, and two copper electrodes (positive/ground). First, the PMMA holder was home made by means of the standard micro-milling process. The detailed drawings of the individual parts of the holder are presented in Figs. S3-S7 (Supporting Information). Next, micro-nozzles (inner diameter $\approx 30 \mu \mathrm{m}$; outer diameter $\approx 50 \mu \mathrm{m}$ ) were made by pulling glass capillaries (outer diameter $\approx 1.5 \mathrm{~mm}$; inner diameter $\approx 0.75 \mathrm{~mm}$; Sutter Instrument) by means of a micropipette puller (P-97, Sutter Instrument). The capillary side of the nozzles was then fixed in the designed channels of the plastic holder using epoxy glue. Then, individual copper wires (outer diameter $\approx 500 \mu \mathrm{m}$ ) were implanted from the top side of the holder to be utilized as electrodes. Lastly, individual counter electrode (copper rings) is attached to complete the integration of the printing head. The distance between the printing nozzle and its integrated ground ring can be adjusted using the designed screw threads.

\section{Experimental set-up}

Figure S8 (Supporting Information) presents the schematic diagram of the home-made EHD inkjet-printing system. Multinozzle print head was positioned above XYZ stage, and for constant power and individual voltage control, it was then linked to a voltage distributor (HM10-5, Hanmac) using individual copper electrodes. The voltage distributer was further connected to a high voltage power supply (10/40A, Trek). For supplying ink to the printing head, the inlet channel of each nozzles was linked to a multi-channel pump (IP-RS232, Ismatec) using Teflon tubes. The jetting behavior of the printing head was observed using a high speed camera (x3, 11X, 5000fps, Motion Pro). Multichannel pump, high speed camera, power supply, and moveable stage (for substrate motion), all were connected using a single modular device (PXI-1042Q, National Instruments), run via a home-built software program created by using LabVIEW.

\section{Surface characterizations}

The structure of the printed circuits was examined by SEM (JSM-7600F, Jeol), AFM (AFM-100, EM4SYS), and optical microscope (BX 41, Olympus). The electrical conductivities of the printed circuits were recorded by using four-probe technique. During the measurement, four electrodes were positioned at the corners of the sample (square area), and the resistance was logged by using a sourcemeter (2450, Keithley). The XRD patterns of silver nanoparticle ink samples were characterized on an X-ray diffractometer (D/MAX 2200 PC, Rigaku).
TABLE 2: Functional inks and their properties.

\begin{tabular}{lccccc}
\hline \hline Inks & $\begin{array}{c}\text { Viscosity } \\
(\mathrm{mPa} \mathrm{s})\end{array}$ & $\begin{array}{c}\text { Surface ten- } \\
\text { sion }(\mathrm{mN} / \mathrm{m})\end{array}$ & Particle size $(\mathrm{nm})$ & $\begin{array}{c}\text { Material } \\
\text { weight (\%) }\end{array}$ & $\begin{array}{c}\text { Curing-condi- } \\
\text { tions (temp/ } \\
\text { time) }\end{array}$ \\
\hline Silver nanoparticle ink & 39 & 5 & 50 & 40 & $250^{\circ} \mathrm{C} / 30 \mathrm{~min}$ \\
Silver organo-metallic ink & 15 & 32 & $\mathrm{~N} / \mathrm{A}$ & 20 & $100^{\circ} \mathrm{C} / 30 \mathrm{~min}$ \\
\hline \hline
\end{tabular}




\section{Acknowledgments}

This work was supported by the Qatar National Research Fund (a member of Qatar Foundation) under Grant NPRP11S-0110-180246. The findings herein reflect the work and are solely the responsibility of the authors.

\section{Funding}

Open access funding provided by the Qatar National Library.

\section{Data availability}

The authors declare that [the/all other] data supporting the findings of this study are available within the article [and its supplementary information files].

\section{Supplementary Information}

The online version contains supplementary material available at https://doi.org/10.1557/s43578-021-00188-4.

\section{Open Access}

This article is licensed under a Creative Commons Attribution 4.0 International License, which permits use, sharing, adaptation, distribution and reproduction in any medium or format, as long as you give appropriate credit to the original author(s) and the source, provide a link to the Creative Commons licence, and indicate if changes were made. The images or other third party material in this article are included in the article's Creative Commons licence, unless indicated otherwise in a credit line to the material. If material is not included in the article's Creative Commons licence and your intended use is not permitted by statutory regulation or exceeds the permitted use, you will need to obtain permission directly from the copyright holder. To view a copy of this licence, visit http://creativecommons.org/ licenses/by/4.0/.

\section{References}

1. M. Gao, L. Li, Y. Song, Inkjet printing wearable electronic devices. J. Mater. Chem. C 5(12), 2971 (2017)

2. A. Khan, J.S. Roo, T. Kraus, J. Steimle, Soft inkjet circuits: rapid multi-material fabrication of soft circuits using a commodity inkjet printer. In: Proceedings of the 32nd Annual ACM Symposium on User Interface Software and Technology, (Association for Computing Machinery, City, 2019), p. 341

3. R.A. Street, W.S. Wong, S.E. Ready, M.L. Chabinyc, A.C. Arias, S. Limb, A. Salleo, R. Lujan, Jet printing flexible displays. Mater. Today. 9(4), 32 (2006)

4. B.-J. de Gans, P.C. Duineveld, U.S. Schubert, Inkjet printing of polymers: state of the art and future developments. Adv. Mater. 16(3), 203 (2004)
5. M. Einat, N. Einat, Two-dimension full array high-speed ink-jet print head. Appl. Phys. Lett. 89(7), 073505 (2006)

6. K.K.B. Hon, L. Li, I.M. Hutchings, Direct writing technologyadvances and developments. CIRP Ann. 57(2), 601 (2008)

7. X. Cao, Y. Ye, Q. Tang, E. Chen, Z. Jiang, J. Pan, T. Guo, Numerical analysis of droplets from multinozzle inkjet printing. J. Phys. Chem. Lett. 11(19), 8442 (2020)

8. H.F. Poon, Electrohydrodynamic printing, Ph.D. Thesis 2002, Princeton University, USA

9. J.-U. Park, M. Hardy, S.J. Kang, K. Barton, K. Adair, D.K. Mukhopadhyay, C.Y. Lee, M.S. Strano, A.G. Alleyne, J.G. Georgiadis, P.M. Ferreira, J.A. Rogers, High-resolution electrohydrodynamic jet printing. Nat. Mater. 6(10), 782 (2007)

10. D.S. Kim, A. Khan, K. Rahman, S. Khan, H.C. Kim, K.H. Choi, Drop-on-demand direct printing of colloidal copper nanoparticles by electrohydrodynamic atomization. Mater. Manuf. Process. 26(9), 1196 (2011)

11. K. Rahman, A. Khan, N.M. Muhammad, J. Jo, K.-H. Choi, Fine-resolution patterning of copper nanoparticles through electrohydrodynamic jet printing. J. Micromech. Microeng. 22(6), 065012 (2012)

12. Y. Han, J. Dong, High-resolution electrohydrodynamic (EHD) direct printing of molten metal. Procedia Manuf. 10, 845 (2017)

13. J. Schneider, P. Rohner, D. Thureja, M. Schmid, P. Galliker, D. Poulikakos, Electrohydrodynamic nanodrip printing of high aspect ratio metal grid transparent electrodes. Adv. Funct. Mater. 26(6), 833 (2016)

14. B. Zhang, J. He, X. Li, F. Xu, D. Li, Micro/nanoscale electrohydrodynamic printing: from 2D to 3D. Nanoscale 8(34), 15376 (2016)

15. Y. Huang, Y. Ding, J. Bian, Y. Su, J. Zhou, Y. Duan, Z. Yin, Hyperstretchable self-powered sensors based on electrohydrodynamically printed, self-similar piezoelectric nano/microfibers. Nano Energy 40, 432 (2017)

16. S. Ali, J. Bae, C.H. Lee, Stretchable photo sensor based on graphene/perylene composite utilizing electrohydrodynamic fabrication technique (SPIE, City, 2015), APO

17. S. Ali, J. Bae, K.H. Choi, C.H. Lee, Y.H. Doh, S. Shin, N.P. Kobayashi, Organic non-volatile memory cell based on resistive elements through electro-hydrodynamic technique. Org. Electron. 17, 121 (2015)

18. R.K.F. Teng, A.A. Mostafa, A. Karim, Study of solar cell fabrication using an electrostatic thick-film printing method. IEEE Trans. Ind. Electron. 37(5), 419 (1990)

19. D. Byun, G. Kang, Z. Bin, Y. Jang, K.S. Jeon, B. Seong, W. Son, V.D. Nguyen, 36-1: invited paper: high-resolution inducedelectrohydrodynamic (iEHD) jet printing for display. SID Symp. Digest Techn Pap. 51(1), 505 (2020)

20. S. Khan, Y.H. Doh, A. Khan, A. Rahman, K.H. Choi, D.S. Kim, Direct patterning and electrospray deposition through EHD 
for fabrication of printed thin film transistors. Curr. Appl. Phys. 11(1, Supplement), S271 (2011)

21. S.Y. Kim, K. Kim, Y.H. Hwang, J. Park, J. Jang, Y. Nam, Y. Kang, M. Kim, H.J. Park, Z. Lee, J. Choi, Y. Kim, S. Jeong, B.S. Bae, J.U. Park, High-resolution electrohydrodynamic inkjet printing of stretchable metal oxide semiconductor transistors with high performance. Nanoscale 8(39), 17113 (2016)

22. E.M. Jung, S.W. Lee and S.H. Kim: Printed ion-gel transistor using electrohydrodynamic (EHD) jet printing process Organic Electronics. 52, 123 (2018).

23. T.T.T. Can, T.C. Nguyen, W.-S. Choi, Patterning of high-viscosity silver paste by an electrohydrodynamic-jet printer for use in TFT applications. Sci. Rep. 9(1), 9180 (2019)

24. H.K. Choi, J.-U. Park, O.O. Park, P.M. Ferreira, J.G. Georgiadis, J.A. Rogers, Scaling laws for jet pulsations associated with high-resolution electrohydrodynamic printing. Appl. Phys. Lett. 92(12), 123109 (2008)

25. K. Shigeta, Y. He, E. Sutanto, S. Kang, A.-P. Le, R.G. Nuzzo, A.G. Alleyne, P.M. Ferreira, Y. Lu, J.A. Rogers, Functional protein microarrays by electrohydrodynamic jet printing. Anal. Chem. 84(22), 10012 (2012)

26. Z. Yin, Y. Huang, N. Bu, X. Wang, Y. Xiong, Inkjet printing for flexible electronics: materials, processes and equipments. Chin Sci. Bull. 55(30), 3383 (2010)

27. M.S. Onses, E. Sutanto, P.M. Ferreira, A.G. Alleyne, J.A. Rogers, Mechanisms, capabilities, and applications of high-resolution electrohydrodynamic jet printing. Small 11(34), 4237 (2015)

28. D. Gao, J. Zhou, Designs and applications of electrohydrodynamic 3D printing. Int. J. Bioprint 5(1), 172 (2019)

29. S.N. Jayasinghe, M.J. Edirisinghe, A novel process for simulataneous printing of multiple tracks from concentrated suspensions. Mater. Res. Innov. 7(2), 62 (2003)

30. B. Quang Tran Si, D. Byun, S. Lee, Experimental and theoretical study of a cone-jet for an electrospray microthruster considering the interference effect in an array of nozzles. J. Aerosol Sci. 38(9), 924 (2007)

31. S.B. Quang Tran, D. Byun, V.D. Nguyen, H.T. Yudistira, M.J. Yu, K.H. Lee, J.U. Kim, Polymer-based electrospray device with multiple nozzles to minimize end effect phenomenon. J. Electrostat. 68(2), 138 (2010)

32. K.-H. Choi, A. Khan, K. Rahman, Y.-H. Doh, D.-S. Kim, K.-R. Kwan, Effects of nozzles array configuration on cross-talk in multi-nozzle electrohydrodynamic inkjet printing head. J. Electrostat. 69(4), 380 (2011)

33. A. Khan, K. Rahman, M.-T. Hyun, D.-S. Kim, K.-H. Choi, Multinozzle electrohydrodynamic inkjet printing of silver colloidal solution for the fabrication of electrically functional microstructures. Appl. Phys. A 104(4), 1113 (2011)

34. A. Khan, K. Rahman, D.S. Kim, K.H. Choi, Direct printing of copper conductive micro-tracks by multi-nozzle electrohydrodynamic inkjet printing process. J. Mater. Process. Technol. 212(3), 700 (2012)

35. Y. Pan, Y. Huang, L. Guo, Y. Ding, Z. Yin, Addressable multinozzle electrohydrodynamic jet printing with high consistency by multi-level voltage method. AIP Adv. 5(4), 047108 (2015)

36. Z. Yin, Y. Huang, Y. Duan, H. Zhang, Nozzles for EHD Printing, in Electrohydrodynamic Direct-Writing for Flexible Electronic Manufacturing (Springer, Singapore, 2018), p. 117

37. J. Zeleny, The electrical discharge from liquid points, and a hydrostatic method of measuring the electric intensity at their surfaces. Phys. Rev. 3(2), 69 (1914)

38. G. Wald, The receptors of human color vision action spectra of three visual pigments in human cones account for normal color vision and color-blindness. Science 145(3636), 1007 (1964)

39. J.D. Regele, M.J. Papac, M.J.A. Rickard, D. Dunn-Rankin, Effects of capillary spacing on EHD spraying from an array of cone jets. J. Aerosol Sci. 33(11), 1471 (2002)

40. A. Khan, S. Lee, T. Jang, Z. Xiong, C. Zhang, J. Tang, L.J. Guo, W.-D. Li, High-performance flexible transparent electrode with an embedded metal mesh fabricated by cost-effective solution process. Small 12(22), 3021 (2016)

41. C. Zhang, A. Khan, J. Cai, C. Liang, Y. Liu, J. Deng, S. Huang, G. Li, W.-D. Li, Stretchable transparent electrodes with solutionprocessed regular metal mesh for an electroluminescent lightemitting film. ACS Appl. Mater. Interfaces 10(24), 21009 (2018)

42. T. Li, Z. Huang, Z. Suo, S.P. Lacour, S. Wagner, Stretchability of thin metal films on elastomer substrates. Appl. Phys. Lett. 85(16), 3435 (2004)

43. J. Zhao, Z. Chi, Z. Yang, X. Chen, M.S. Arnold, Y. Zhang, J. Xu, Z. Chi, M.P. Aldred, Recent developments of truly stretchable thin film electronic and optoelectronic devices. Nanoscale 10(13), 5764 (2018)

44. A. Khan, C. Liang, Y.-T. Huang, C. Zhang, J. Cai, S.-P. Feng, W.-D. Li, Template-electrodeposited and imprint-transferred microscale metal-mesh transparent electrodes for flexible and stretchable electronics . Adv. Eng. Mater. 21(12), 1900723 (2019)

45. J. Lee, S. Chung, H. Song, S. Kim, Y. Hong, Lateral-crack-free, buckled, inkjet-printed silver electrodes on highly pre-stretched elastomeric substrates. J. Phys. D Appl Phys. 46(10), 105305 (2013)

46. J. Byun, B. Lee, E. Oh, H. Kim, S. Kim, S. Lee, Y. Hong, Fully printable, strain-engineered electronic wrap for customizable soft electronics. Sci. Rep. 7(1), 45328 (2017)

47. J.M. Abu-Khalaf, L. Al-Ghussain, A.A. Al-Halhouli, Fabrication of stretchable circuits on polydimethylsiloxane (PDMS) pre-stretched substrates by inkjet printing silver nanoparticles. Materials 11(12), 2377 (2018)

48. S. Chung, J. Lee, H. Song, S. Kim, J. Jeong, Y. Hong, Inkjetprinted stretchable silver electrode on wave structured elastomeric substrate. Appl. Phys. Lett. 98(15), 153110 (2011) 
49. T. Liimatta, E. Halonen, H. Sillanpää, J. Niittynen, M. Mäntysalo, Inkjet printing in manufacturing of stretchable interconnects. In: Proceedings of the 2014 IEEE 64th Electronic Components and Technology Conference (ECTC), (City, 2014), p. 151

50. R.C. Webb, Y. Ma, S. Krishnan, Y. Li, S. Yoon, X. Guo, X. Feng, Y. Shi, M. Seidel, N.H. Cho, J. Kurniawan, J. Ahad, N. Sheth, J. Kim, J.G. Taylor, T. Darlington, K. Chang, W. Huang, J. Ayers, A. Gruebele, R.M. Pielak, M.J. Slepian, Y. Huang, A.M. Gorbach, J.A. Rogers, Epidermal devices for noninvasive, precise, and continuous mapping of macrovascular and microvascular blood flow. Sci. Adv. 1(9), e1500701 (2015)

51. J.C. Yang, J. Mun, S.Y. Kwon, S. Park, Z. Bao, S. Park, Electronic skin: recent progress and future prospects for skin-attachable devices for health monitoring robotics, and prosthetics. Adv. Mater. 31(48), 1904765 (2019)

52. X. Wu, Y. Han, X. Zhang, C. Lu, Highly sensitive, stretchable, and wash-durable strain sensor based on ultrathin conductive layer@ polyurethane yarn for tiny motion monitoring. ACS Appl. Mater. Interfaces 8(15), 9936 (2016)
53. S. Ali, S. Khan, A. Khan, A. Bermak, Developing conductive fabric threads for human respiratory rate monitoring. IEEE Sens. J. 21, 4350-4356 (2020)

54. A.S. Nittala, A. Khan, K. Kruttwig, T. Kraus, J. Steimle, PhysioSkin: rapid fabrication of skin-conformal physiological interfaces. in Proceedings of the 2020 CHI Conference on Human Factors in Computing Systems, (Association for Computing Machinery, City, 2020), p. 1

55. H. Souri, H. Banerjee, A. Jusufi, N. Radacsi, A.A. Stokes, I. Park, M. Sitti, M. Amjadi, Wearable and stretchable strain sensors: materials sensing mechanisms, and applications. Adv. Intell. Syst. 2(8), 2000039 (2020)

56. C. Yan, J. Wang, W. Kang, M. Cui, X. Wang, C.Y. Foo, K.J. Chee, P.S. Lee, Highly stretchable piezoresistive graphene-nanocellulose nanopaper for strain sensors. Adv. Mater. 26(13), 2022 (2014) 